\title{
Simulación del comportamiento de motores monofásicos de inducción
}

\section{(Simulation of the behavior of single-phase induction motors)}

Gustavo J. Boschi, i Guillermo A. Molina,ii Luis A. S. Carrazán ${ }^{i i i}$

\& Mariano Deleuiv

\section{Resumen}

El presente documento expone una fase de la investigación de un proyecto llamado "Contactor inteligente para el ahorro de energía" [3] el cual se aplica a motores de inducción de corriente alterna. El propósito del presente reporte es aplicar los modelos circuitales del motor de inducción, adaptándolos al programa de simulación a utilizar para el posterior análisis de factibilidad de circuitos de control propuestos. Esta metodología permite ensayar los modelos de control propuestos para la alimentación de motores de inducción sin la necesidad de desarrollar hardware durante la etapa de evaluación de ideas.

Palabras clave: modelo circuital, simulación, control de potencia.

Facultad de Ingeniería, Universidad de la Marina Mercante, Buenos Aires, Argentina, email: gustavo.boschi@docentes.udemm.edu.ar

ii Facultad de Ingeniería, Universidad de la Marina Mercante, Buenos Aires, Argentina, email: gmolina@udemm.edu.ar

iii Facultad de Ingeniería, Universidad de la Marina Mercante, Buenos Aires, Argentina, email: luis.carrazan@docentes.udemm.edu.ar

iv Facultad de Ingeniería, Universidad de la Marina Mercante, Buenos Aires, Argentina, email: mariano.deleu@docentes.udemm.edu.ar 


\section{Abstract}

This document presents a phase of the investigation of a project called "Intelligent Contactor for Energy Saving" [3] which is applied to AC induction motors. The purpose of this report is to apply the induction motor circuit models, adapting them to the simulation program to be used for the subsequent feasibility analysis of proposed control circuits. This methodology allows testing the control models proposed for the induction motor power supply without the need to develop hardware during the idea evaluation stage.

Keywords: circuit model, simulation, power control. 


\section{Introducción}

Los motores de inducción de corriente alterna son dispositivos muy utilizados en aplicaciones que requieren una velocidad de operación constante. Con ciertas tecnologías modernas esta tendencia fue cambiando en los últimos años y por medio de inverters es posible encontrar motores de inducción de corriente alterna en aplicaciones que requieren velocidad variable en reemplazo de motores de corriente continua. En general el motor de inducción es más económico y de fácil mantenimiento si se lo compara con otras alternativas como por ejemplo el ya mencionado motor de corriente continua. [2]

Dentro de los motores de inducción encontramos los del tipo monofásicos, los cuales encuentran un notable uso en hogares, en aplicaciones como heladeras, ventiladores de techo, aspiradoras, equipos de aire acondicionado, etc.

\section{Metodología}

Como antes se mencionó, el presente trabajo tiene como finalidad adaptar para la simulación un modelo eléctrico que caracterice el funcionamiento del motor con el propósito de predecir su comportamiento ante la aplicación de distintos tipos de señales de alimentación, para lo cual se seguirá el siguiente lineamiento:

- Revisión del principio de funcionamiento y características de un motor de inducción.

- Explicación del circuito eléctrico equivalente del motor asincrónico.

- Implementación del circuito eléctrico equivalente en el programa de simulación.

\subsection{Principio de funcionamiento del motor de inducción monofásico:}

En este tipo de motor, al existir en él una sola fase, solo puede generarse un campo que varía entre dos polos, careciendo de campo rotatorio. En esta situación el rotor no tendrá un par inducido, permaneciendo detenido y presentando una condición prácticamente de cortocircuito en el bobinado. Lo anteriormente descripto trae algunas observaciones, entre las que podemos del estator. Si se logra dar un primer impulso al rotor, éste comenzará a girar dado que se inducirá un par, lo que provocará salir gradualmente de la condición de cortocircuito destacar [2]:

- Según sea el sentido del primer impulso del rotor, ese será el giro de funcionamiento en régimen.

- La corriente inicial de arranque es muy superior a la corriente de trabajo.

- Es necesario algún medio que permita el arranque automático y asegure el sentido del giro del motor. 
Con la finalidad de tener un arranque con sentido de giro definido y evitar el cortocircuito inicial del bobinado estatórico, los mencionados motores se fabrican con un bobinado adicional en el estator denominado bobinado auxiliar.

Para lograrlo, se requiere que este bobinado esté desfasado mecánica y eléctricamente respecto del bobinado principal. Para producir el desfasaje eléctrico se coloca un capacitor en serie en dicho bobinado auxiliar.

De esta manera se logra conseguir un campo magnético giratorio en torno al eje del rotor. Este campo magnético induce corrientes eléctricas en las barras del rotor, dichas corrientes a su vez generan un campo propio en oposición al que le dio origen. La interacción de ambos campos da lugar a un par inducido en el rotor, produciendo la rotación del mismo.

\subsection{Parámetros característicos del motor asincrónico:}

\section{Velocidad sincrónica:}

La velocidad con la que gira el campo magnético en el estator, que determinará la velocidad de giro del rotor, se llama velocidad sincrónica (SS). La velocidad SS es función de la frecuencia de línea y del número de polos tal como se ve en la ecuación $1[1]$.

$$
S S[r p m]=\frac{120 \times f}{p}
$$

Siendo:

$\mathrm{f}=$ frecuencia de línea (en general 50 o $60 \mathrm{~Hz}$ ).

$\mathrm{p}=$ número de polos del estator, $2,4,8$.

\section{Deslizamiento del motor:}

Para el rotor, no es posible alcanzar la velocidad sincrónica (SS), debido a que el campo estatórico no induciría corriente en las barras del rotor.

Debe entonces existir una diferencia entre sus velocidades.

La relación entre la velocidad del rotor (RS) y la velocidad sincrónica (SS) se denomina deslizamiento y se expresa generalmente en tanto por ciento. [1]

$$
\% S=\frac{(S S-R S) \times 100}{S S}
$$

Donde:

$\% S=$ porcentaje de deslizamiento

$S S=$ velocidad del campo del estator, velocidad sincrónica en rpm.

$R S=$ velocidad de giro del rotor en rpm. 
Si el rotor por algún motivo llegara a igualar la velocidad sincrónica (SS) no podría mantenerla ya que en ese caso no se induciría corriente en las barras del mismo.

En general el deslizamientose encuentra en valores del 2 a $6 \%$.

\section{Torque y tiempo de arranque:}

Las cuestiones referidas al arranque del motor, están sujetas al tipo de motor, su correspondiente "torque operacional" $(\mathrm{CM})$ y a la carga conectada que determina el "torque de carga" (CL), como muestra la figura 1:



Figura 1: variación del torque en función de la velocidad angular.

Siendo:

$\mathrm{Ca}=\mathrm{CM}-\mathrm{CL}$, el torque disponible.

El Ca debe ser suficiente, para que el arranque no sea demasiado lento, pues recalentaría el bobinado del motor; por otro lado, dicho torque no debe ser demasiado elevado pues produciría un esfuerzo innecesario en las juntas o agarres del mismo.

Ca es entonces el torque de aceleración del motor, mientras este exista, el motor acelerará.

Cuando la cupla en la carga (CL) iguale a la cupla del motor (CM) será esta la condición que sitúe al conjunto motor-carga en un modo de trabajo estable, resultando una velocidad (nr) constante [2].

El tiempo de arranque del motor "ta" puede ser relacionado con la cupla disponible, inercia y rozamiento mediante la ecuación 3 [4]:

$$
t a=\int_{0}^{n} \frac{2 \pi \times(J m-J)}{60 \times(C M-C L)} d n
$$


Donde:

ta $=$ tiempo de aceleración hasta la velocidad final [s]

$\mathrm{nr}=$ velocidad final del motor $[\mathrm{rpm}]$

$\mathrm{CM}=$ torque del motor [N.m]

$\mathrm{CL}=$ torque de la carga $[\mathrm{N} . \mathrm{m}]$

$\mathrm{Jm}=$ inercia del motor [kg.m2]

$\mathrm{Jl}=$ inercia de la carga [kg.m2]

Si bien la ecuación 3 brinda una perfecta estimación del ta, requiere múltiples parámetros de fabricación que no son prácticos a la hora de completar un modelo circuital que represente el transitorio de arranque.

Posteriormente se realizarán algunas simplificaciones sobre este tema.

\subsection{Modelo circuital eléctrico del motor de inducción:}

Para el estudio del comportamiento del motor de inducción se utilizará el modelo eléctrico del transformador adaptado al motor (circuito Steinmetz) [1].

El mencionado modelo presenta una geometría de circuito tipo "T" la cual es completamente viable para herramientas de análisis de circuitos utilizados generalmente en la rama en la Ingeniería Eléctrica y/o Electrónica.

Para interpretar el funcionamiento del motor a través del circuito equivalente Steinmetz, la filosofía utilizada es compararlo con un modelo o circuito equivalente de un transformador, donde los bobinados del estator del motor se interpretan como el bobinado primario del transformador y los del rotor como el secundario; en tanto el esfuerzo sobre el eje es el equivalente a una carga colocada en el secundario del transformador. Entonces comparando efectos en el eje del motor, respecto de efectos en el circuito de secundario del transformador, tenemos las siguientes interpretaciones [1]:

Tabla 1: tipos de ensayos a realizar en un motor asincrónico y su relación con el circuito equivalente de un transformador.

\begin{tabular}{|l|l|l|}
\hline Motor & Modelo Transformador & Interpretación \\
\hline Sin Carga en el eje & Sin carga en el secundario & $\begin{array}{l}\text { Representa las pérdidas del } \\
\text { motor (vacío) }\end{array}$ \\
\hline Con carga en el eje & Con carga en el secundario. & $\begin{array}{l}\text { Funcionamiento en determinada } \\
\text { condición de carga }\end{array}$ \\
\hline Rotor bloqueado & Secundario en cortocircuito. & $\begin{array}{l}\text { Condición de arranque del motor } \\
\text { (reposo) }\end{array}$ \\
\hline
\end{tabular}


Por otro lado, como estamos interesados en saber que sucede visto del lado del primario, se reflejan los resistores e inductores desde el secundario al circuito primario, como muestra la figura 2 [1]:



Figura 2: circuito equivalente del motor referido al primario

Donde:

Rs $=$ resistencia del bobinado del estator.

$\mathrm{Xs}=$ reactancia de dispersión del estator.

$\mathrm{Rr}=$ resistencia del rotor reflejada al circuito primario.

$\mathrm{Xr}=$ reactancia de dispersión del rotor reflejada al circuito primario.

$\mathrm{Rp}=$ resistencia de pérdidas en el núcleo.

$\mathrm{Xm}=$ reactancia de Magnetización.

\section{Potencia Eléctrica y Mecánica:}

Es simple interpretar, utilizando el modelo Steinmetz (figura 2), que la potencia de pérdidas y la convertida por el motor en energía mecánica están representadas por siguientes resistores [1]:

$\mathrm{Rs} ; \mathrm{Rr} ; \mathrm{Rp}$ y

Del análisis de la figura 2 se pone en evidencia que Rs, Rr y Rp representan potencia de pérdidas.La potencia útil en el eje viene dada por la siguiente expresión[1]:

$$
\text { Pmec }=\frac{1-s}{s} R r|i r|^{2}
$$

Tomando en cuenta que la potencia es igual al Torque por la velocidad angular, resulta que la cupla motora es [1]:

$$
C M=\frac{P m e c}{w}=\frac{1-s}{s w} R r|i r|^{2}
$$




\section{Desarrollo}

\subsection{Relevamiento de parámetros:}

Con la finalidad de contar con un modelo circuital (modelo Steinmetz), para las posteriores simulaciones, es necesario realizar ensayos al motor de estudio del presente trabajo que posee las características de la tabla 2.

Las características de dicho motor son:

Tabla 2: características mecánicas y eléctricas del motor en estudio

\begin{tabular}{|l|l|l|l|}
\hline Motor Marca & $\begin{array}{l}\text { Potencia } \\
\text { Nominal }\end{array}$ & $\begin{array}{l}\text { Velocidad De } \\
\text { Rotacion }\end{array}$ & $\begin{array}{l}\text { Corriente } \\
\text { Nominal }\end{array}$ \\
\hline $\begin{array}{l}\text { Czerweny } \\
\text { monofásico }\end{array}$ & $0,5 \mathrm{CV}$ & $2830 \mathrm{rpm}$ & $2.1 \mathrm{~A}$ \\
\hline
\end{tabular}

Los parámetros del modelo Steinmetz a relevar están resaltados en círculos en la figura 3.

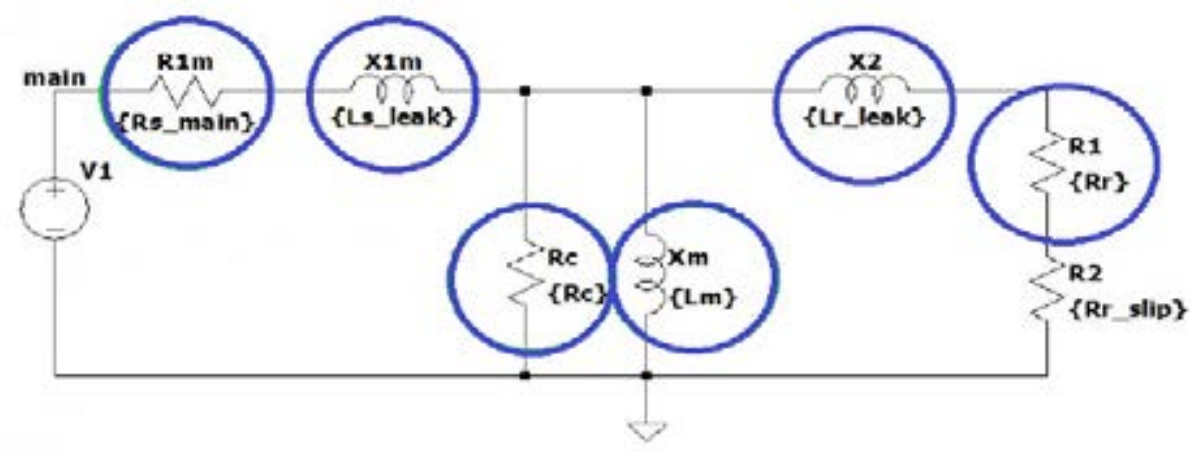

Figura 3: parámetros a obtener del circuito equivalente

Brevemente se describirá como se obtuvieron los mencionados parámetros:

Midiendo con un puente de Wheatstone en el bobinado de trabajo se obtuvo:

$\mathrm{R} 1 \mathrm{~m}=2 \Omega$.

Del ensayo a rotor desbloqueado $(\mathrm{R} 2=\infty)$ surge que:

$\mathrm{Rc}=2000 \Omega$

$\mathrm{Lm}=1 \mathrm{H}$

Del ensayo a rotor bloqueado $(\mathrm{R} 2=0)$ se obtuvo:

Ls_leak $=$ Lr_leak $=30 \mathrm{mH}$

$\mathrm{Rr}=7 \Omega$ 
Habiendo relevado todos los parámetros, el modelo circuital preparado para la simulación queda como muestra la figura 4 :

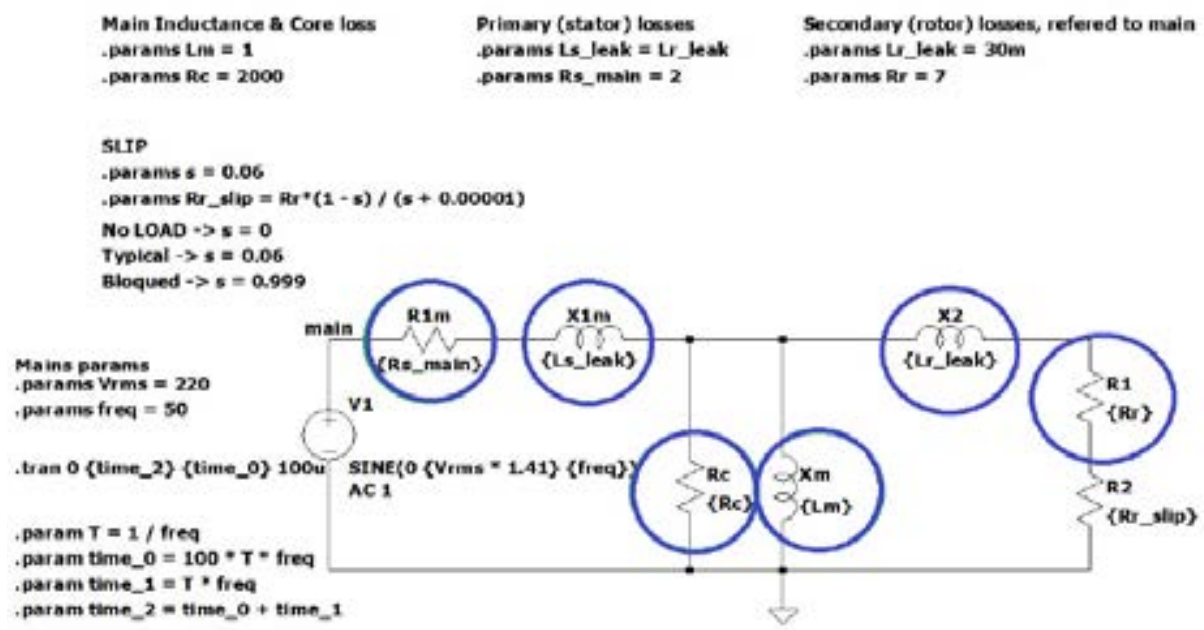

Figura 4: circuito eléctrico preparado para realizar la simulación.

3.2 Adaptación del modelo circuital a la simulación:

Para el desarrollo de las simulaciones se utilizará el programa LTspice, que es un software de computadoras de uso libre y que implementa la simulación de circuitos electrónicos por medio de SPICE, Simulation Program with Integrated Circuit Emphasis - Computer Analisys of Nonlinear Circuits, Excluding Radiation.

\subsubsection{Modelo Simple de simulación:}

El modelo del transformador de Steinmetz supone un régimen permanente en estado estable del conjunto motor-carga [1]. Esto significa que el valor de deslizamiento es constante. Sin embargo, como parte de este desarrollo, se necesita conocer que sucede en períodos transitorios con pequeñas variaciones del deslizamiento dentro o alrededor de su punto de trabajo.

Se comenzará validando un modelo simple con datos extraídos del motor y luego se incrementara la complejidad del modelo para reflejar los mencionados estados transitorios. La figura 5 muestra lo antes mencionado. 


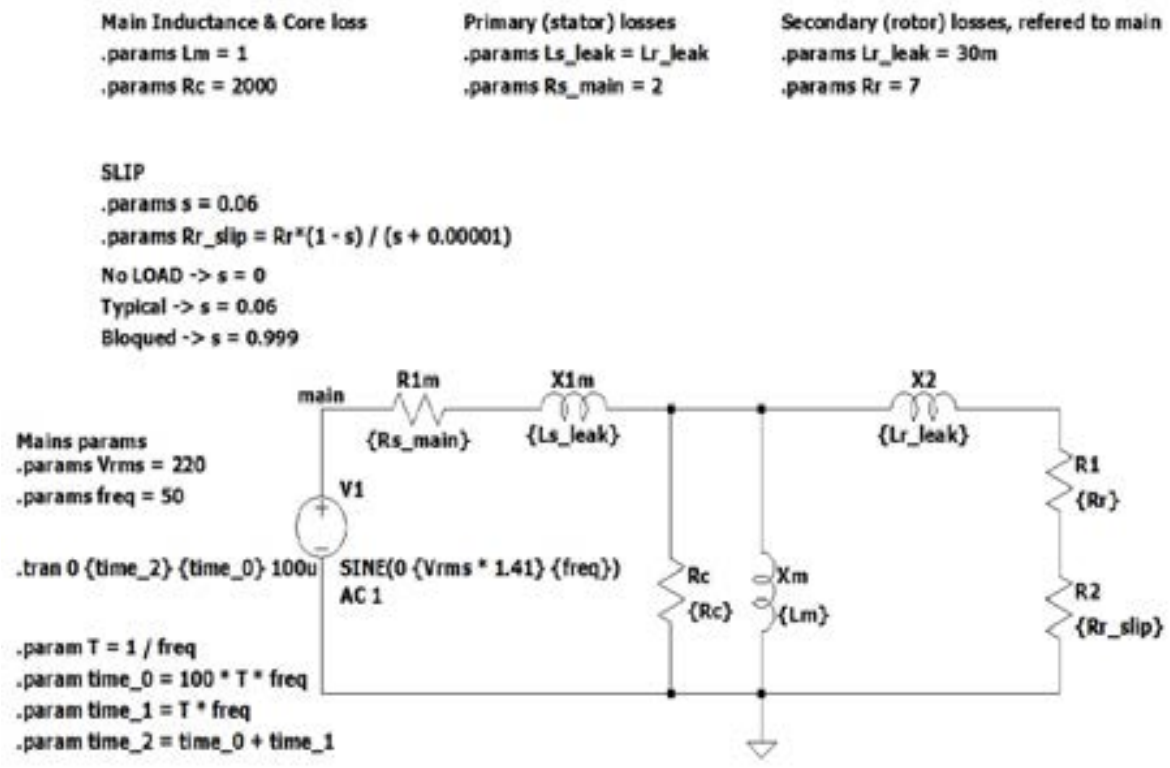

Figura 5: circuito eléctrico para simular una carga fija.

\subsubsection{Modelo ampliado con dependencia del deslizamiento- Modelo Estático}

Como necesitamos evaluar estados transitorios, es importante que las magnitudes del modelo puedan variar en función del deslizamiento. El deslizamiento hace que el valor del resistor $\left(\frac{1-s}{s}\right)$. Rr del modelo de la figura 5 se vea modificado conforme el deslizamiento varia.

Por lo tanto para lograr un circuito que contemple dicha variación en el tiempo y pueda adaptarse al programa de simulación, es necesario convertir dicho resistor (dependiente del deslizamiento) en un resistor dinámico también con dependencia del deslizamiento.

Para emular tal resistor, lo modelaremos colocando dos fuentes de tensión ideales, una con un potencial de $0 \mathrm{~V}$ que nos permitirá medir la corriente que la atraviesa y al tener potencial de $0 \mathrm{~V}$ no generará cambios al circuito original. La segunda es una fuente de tensión controlada por la corriente de la primera fuente. Finalmente con el conjunto de ambas fuentes colocadas en serie, se consigue una tensión que varia en función de la corriente que la atraviesa. Si esta variación es lineal y directamente proporcional, dicho conjunto se convierte en una resistencia.

Por ultimo agregaremos una nueva variable a la fuente controlada (fuente V2 de la figura 6) que representará el deslizamiento. Nos queda entonces un resistor variable en función de esta última fuente. 
El modelo queda como muestra figura 6:

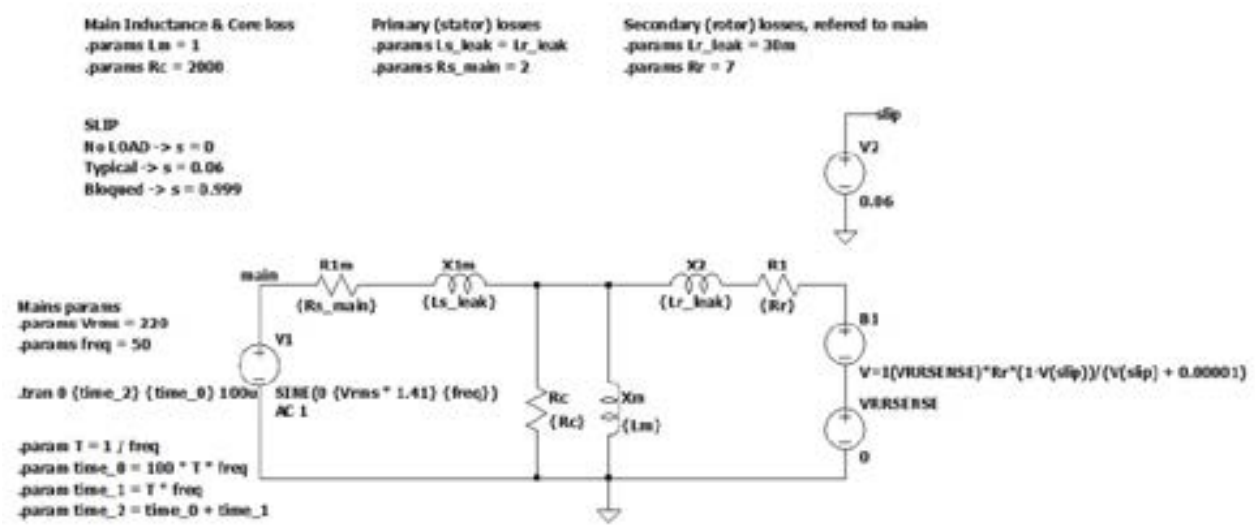

Figura 6: emulación de carga variable en el circuito utilizado para la simulación.

En este modelo el valor de deslizamiento ahora se modela como una fuente de tensión de entre 0 y $1 \mathrm{~V}$ (recordar que el deslizamiento es una magnitud adimensional que varía entre 0 y 1$)$.

3.2.3 Modelo ampliado con dependencia del deslizamiento y tiempo de arranque - Modelo Dinámico:

Como se explicó en 2.1, ecuación 3; el tiempo de arranque o transitorio ta, depende mayormente del torque disponible $\mathrm{Ca}$. Para no complejizar demasiado el modelo y teniendo en cuenta que los sistemas reales, en general presentan respuestas exponenciales en el tiempo de la forma $e^{x} 01-e^{x}$, se propone un circuito simple de primer orden para el arranque. Se suponen 20 segundos de intervalo de arranque, situación bastante típica en este tipo de motores. 
El modelo circuital implementado en el simulador para emular dicha dependencia temporal se muestra en la siguiente figura 7 :

\begin{tabular}{|c|c|c|}
\hline $\begin{array}{l}\text { Lan Indictance } \mathrm{k} \text { Core loss } \\
\text { perans } 1 \mathrm{n}=1 \\
\text { parans } \mathrm{RC}=2000\end{array}$ & 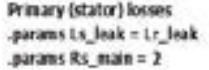 & $\begin{array}{l}\text { Secondery (rota) bsses, retered to naie } \\
\text { paramstr_leak }=30 \mathrm{~m} \\
\text { parans } k r=\gamma\end{array}$ \\
\hline
\end{tabular}

SIP

NotoAD $\rightarrow s=0$

Troial $\rightarrow s=\cos$

Blocued $\rightarrow s=0.595$

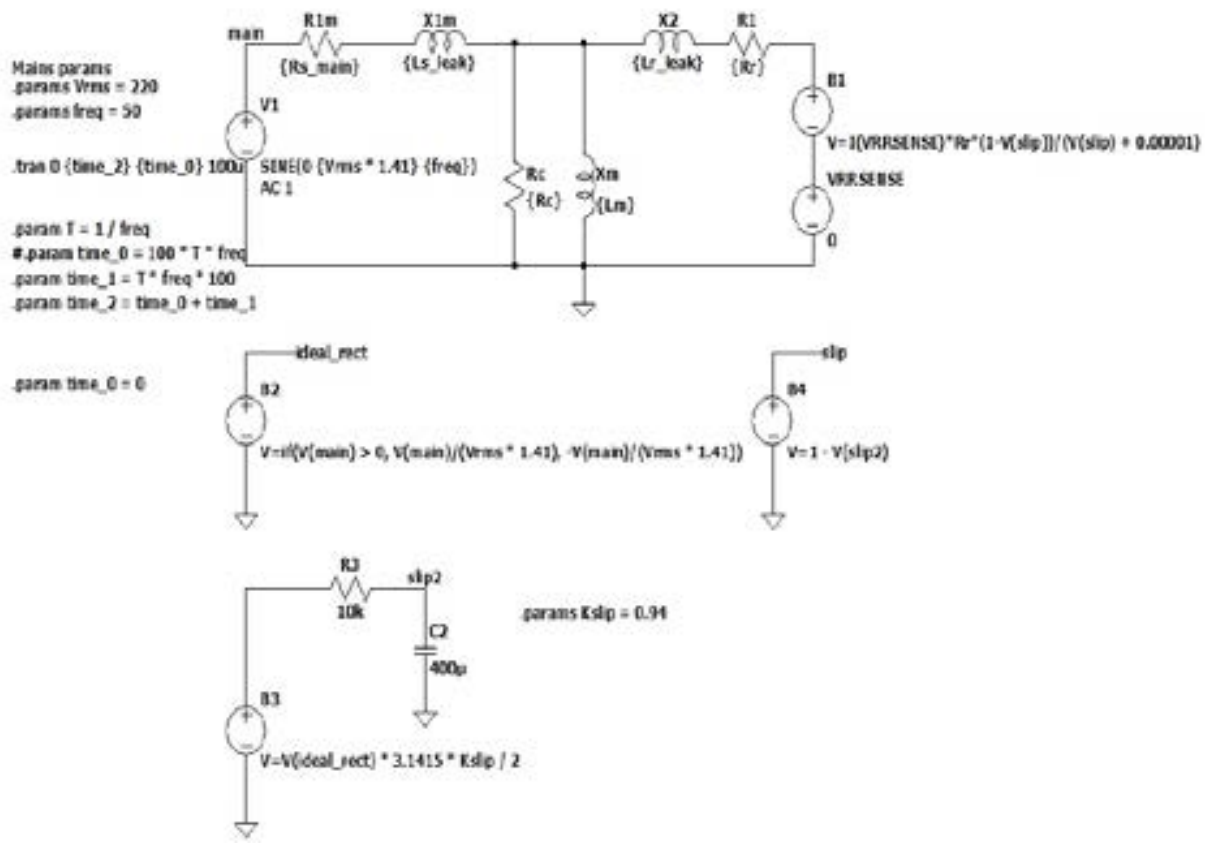

Figura 7: circuito utilizado para la simulación del comportamiento dinámico.

\section{Pruebas realizadas}

A los efectos de comprobar la validez de los modelos desarrollados y verificar su validez con el comportamiento real del mencionado modelo de motor ser realizaron las simulaciones que a continuación se detallan:

\section{Simulación del Modelo Simple:}

Se cargó en el simulador el circuito con los parámetros de la figura 5 (ítem 3.2.1), obteniéndose los siguientes resultados: 


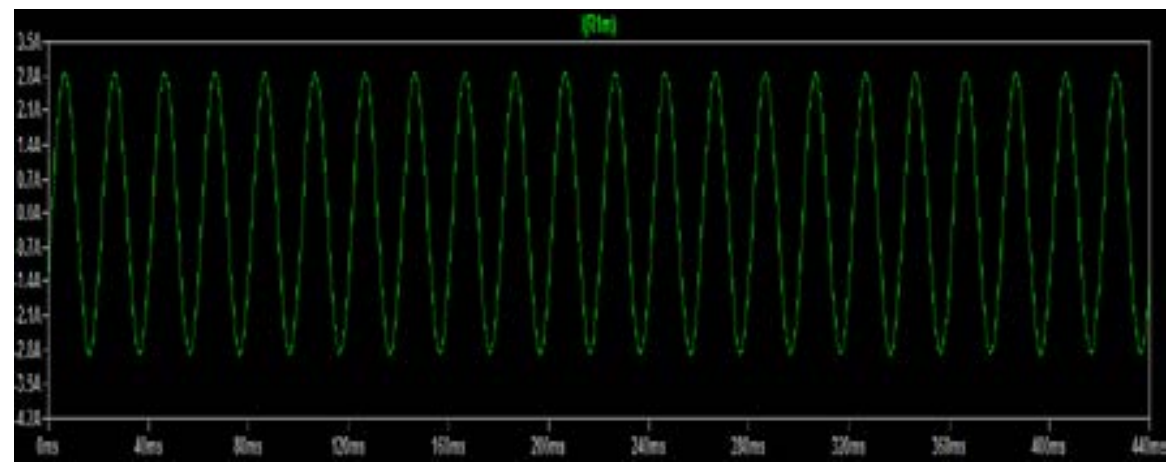

Figura 8: señal corriente para una carga constante en el tiempo.

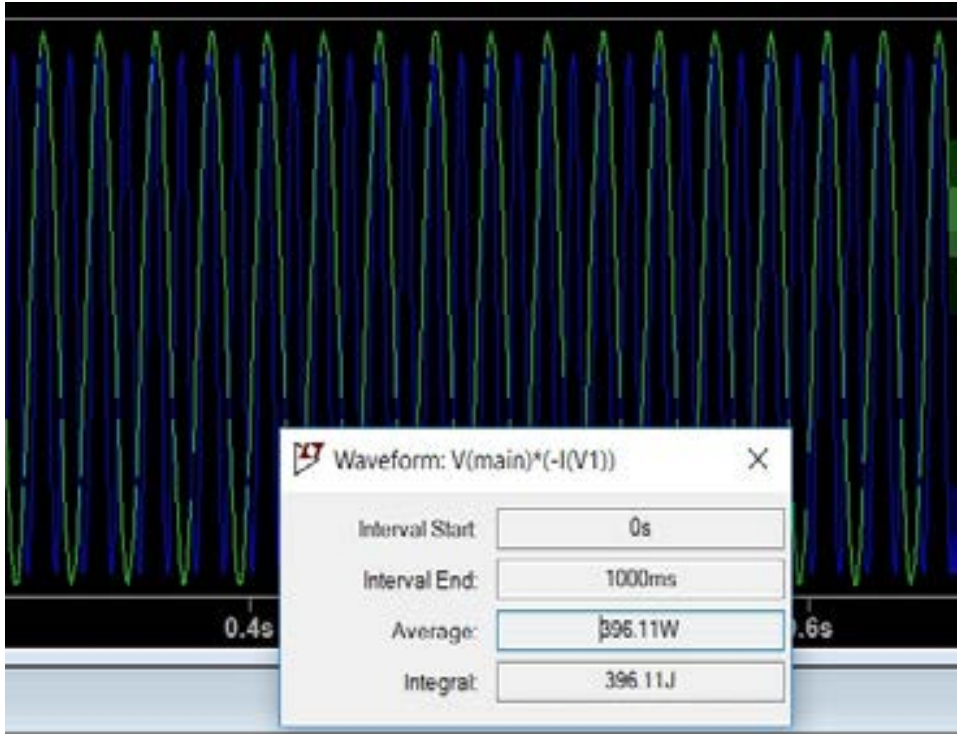

Figura 9: obtención de la potencia media consumida por el motor.

Puede observarse:

- Figura 8: la forma de la señal corriente tiene una amplitud constante, pues en este modelo el deslizamiento es constante.

- Figura 8: el valor pico de la corriente es aproximadamente de 2,8 A, hecho que se corresponde con la corriente eficaz de 2,12 A del motor en cuestión, según la tabla 2.

- En la figura 9: la potencia consumida por el motor es de 396,11 W, valor similar al de la tabla 2 (características del motor en estudio).

Nota: en dicha figura la señal azul es la potencia instantánea, mientras que la verde es la corriente que toma de la fuente de alimentación. 


\section{Simulación del modelo dinámico:}

Se cargó en el simulador el circuito con los parámetros de la figura 7 (ítem 3.2.3), obteniéndose los siguientes resultados:

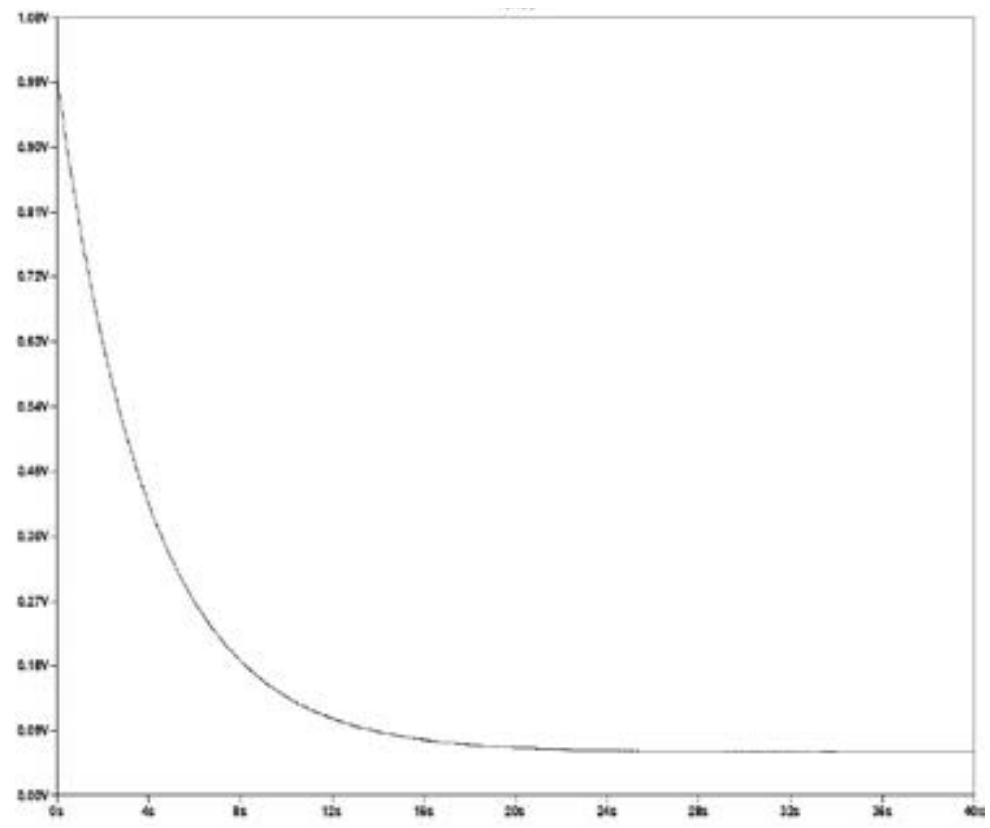

Figura 10: variación del deslizamiento en función del tiempo

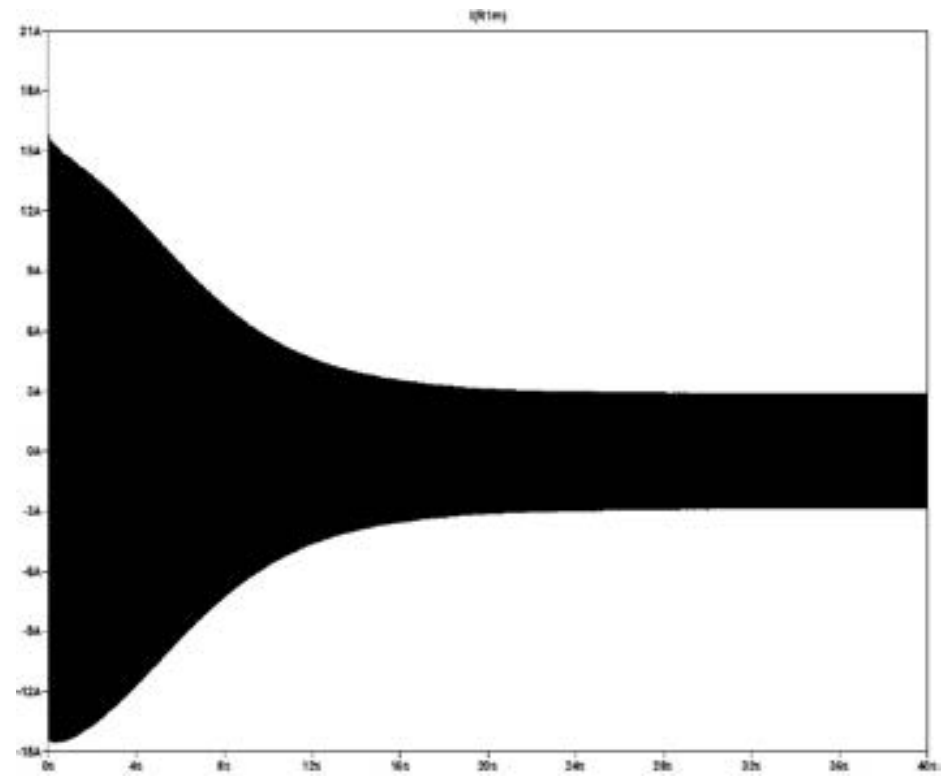

Figura 11: envolvente de la corriente de arranque del motor 
Puede observarse:

- Figura 10: representa la variación del deslizamiento "s" en función del tiempo, como hipótesis simplificativa la variación de "s" en función del tiempo es del tipo exponencial, tal como se dijo en el apartado ítem 3.2.3. En dicha figura "s" está representado por una tensión que podrá variar de $1 \mathrm{~V}$ a $0 \mathrm{~V}$ (tener en cuenta que "s" coincide numéricamente con dicha tensión).

- Figura 11: muestra las envolventes (superior e inferior) de los picos de la señal corriente de alimentación del motor. Este transitorio de la corriente está en concordancia con el de los motores reales.

El valor pico de la corriente de régimen permanente se aproxima a los 2,8 A, hecho que se corresponde con la corriente eficaz de 2,12 A del motor en cuestión, según la tabla 2.

\section{Obtención de la curva de arranque en el motor real:}

Para obtener la variación de la corriente en función del tiempo, se conectó en serie a la línea de alimentación del motor una resistencia de bajo valor con la finalidad de que la medición no altere el funcionamiento normal del motor. Sobre dicha resistencia se conectó la entrada de un canal del osciloscopio [5].

Como carga mecánica al motor, se le colocó un ventilador que impele aire en una cañería de un sistema experimental de ventilación [3].

Por otro lado para efectuar la medición con el osciloscopio, respetando las normas de seguridad de puesta a tierra de este último, el motor fue alimentado mediante un transformador de aislación galvánica. La figura 12 muestra lo antes descripto.

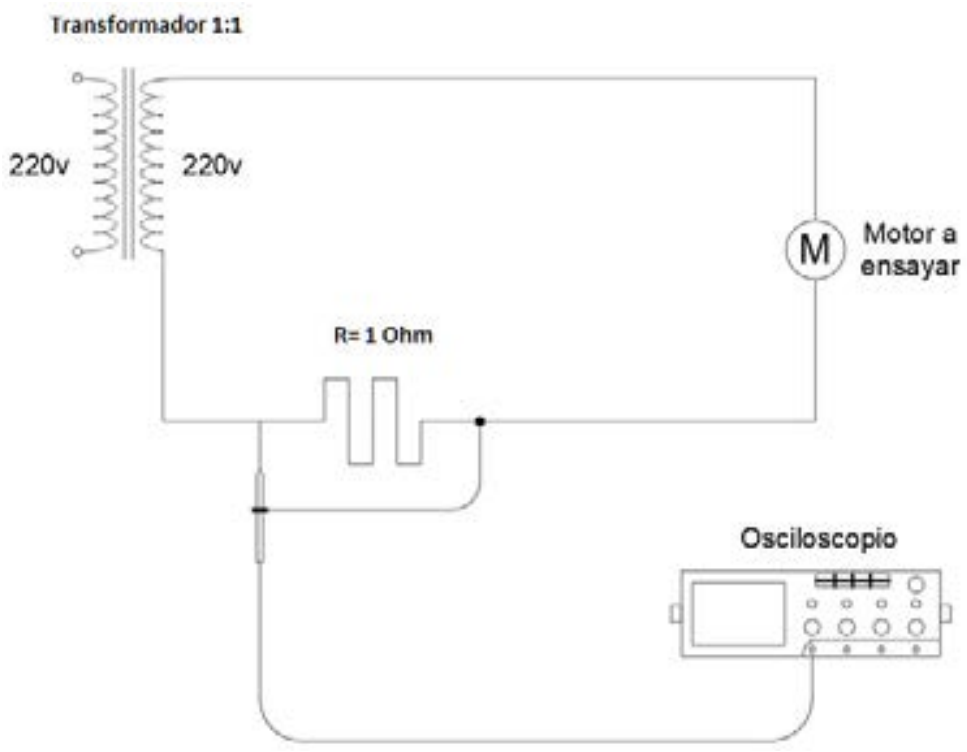

Figura 12: forma de obtener la corriente en función del tiempo 
El osciloscopio fue utilizado como adquisidor de datos, suministrando los mismos en un archivo del tipo ".CSV".

Habiéndose procesado de manera adecuada dichos datos en una planilla Excel, se obtuvo el gráfico en el cual se representa las envolventes de los picos de la señal corriente en el tiempo, tal como lo muestra la figura 13:



Figura 13: envolvente real de la corriente de arranque del motor

\section{Observaciones:}

Pueden apreciarse las similitudes en los valores de corrientes pico de arranque y de régimen permanente respecto a la gráfica de la figura 11. En cuanto al tiempo de entrada en régimen permanente puede observarse que el ensayo al motor indica que se encuentra aproximadamente a partir de los 15 segundos, mientras que en la simulación se encuentra a partir de los 20 segundos. Cabe aclarar que en este último caso no fue considerada - para el desarrollo- el tipo de carga, sino una hipótesis simplificativa de 20 segundos para alcanzar el régimen permanente (parágrafo 3.2.3) para la potencia nominal del motor.

Por último se observa que ambas curvas envolventes tienen la forma del tipo exponencial decreciente.

\section{Conclusión}

El modelo dinámico, responde coherentemente con los datos característicos del motor, pudiendo entonces ser validado tanto con los valores de corriente como de potencia en régimen permanente. Por otro lado se validó la forma de la curva del transitorio de corriente de arranque con un ensayo real, arrojando valores próximos entre ambas. 
Es ahora viable probar los circuitos de control que se quieran proyectar antes de pasar a la construcción de un prototipo.

Por último un modelo que represente bien a un sistema ahorrará tiempo de manera muy significativa en ensayos, pruebas y corrección de errores en la etapa de desarrollo de un proyecto.

\section{Referencias}

Chapman, S., Máquinas Eléctricas (2a. ed.), Santafé de Bogotá, Colombia: McGraw-Hill Interamericana (1993).

Maloney, T.: Electrónica industrial moderna (5a. Ed.). México, D.F., México: Pearson (2006).

Boschi, G., Molina, G., Carrazán L., Deleu M.: Programador Lógico para control de potencia. Buenos Aires, Argentina: 47 JAIIO (2018).

Gray, A.: Máquinas Eléctricas (3a. ed.), Buenos Aires, Argentina: Eudeba (1977).

Müller, Hörnemann, Hübscher, Jagla, Larisch, Pauly: Electrotecnia de potencia, Barcelona, España: Ed. Reverté (1994). 
